ÄSTHETIK DES SCHEITERNS 
Michael Kämper-van den Boogaart

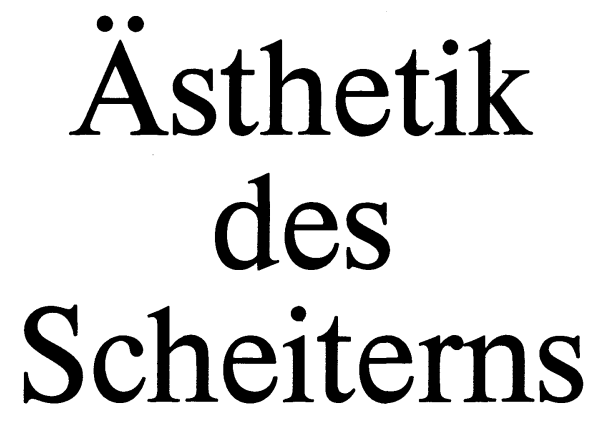

Studien zu Erzähltexten von Botho Strauß, Jürgen Theobaldy, Uwe Timm u. a. 


\section{METZLER STUDIENAUSGABE}

Die Deutsche Bibliothek - CIP-Einheitsaufnahme

\section{Kämper-van den Boogaart, Michael:}

Ästhetik des Scheiterns : Studien zu Erzähltexten von Botho

Strauß, Jürgen Theobaldy, Uwe Timm u.a. / Michael Kämper-

van den Boogaart. -Stuttgart : Metzler, 1992

(Metzler Studienausgabe)

ISBN 978-3-476-00857-2

ISBN 978-3-476-00857-2

ISBN 978-3-476-03430-4 (eBook)

DOI 10.1007/978-3-476-03430-4

Dieses Werk einschließlich aller seiner Teile ist urheberrechtlich geschützt. Jede Verwertung außerhalb der engen Grenzen des Urheberrechtsgesetzes ist ohne Zustimmung des Verlages unzulässig und strafbar. Das gilt insbesondere für Vervielfältigungen, Übersetzungen, Mikroverfilmungen und die Einspeicherung und Verarbeitung in elektronischen Systemen.

(C) 1992 Springer-Verlag GmbH Deutschland

Ursprünglich erschienen bei J. B. Metzlersche Verlagsbuchhandlung und Carl Ernst Poeschel Verlag GmbH in Stuttgart 1992 
Inhalt

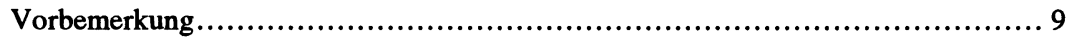

1. Sinn: verlustig .................................................................. 13

Krisengerede: Das Ende der Dekade ist die Stunde der Kommentatoren...........13

Big Sinn ist tot. Zur Historizität substantiellen Sinns............................ 17

2. Facetten der Melancholie................................................25

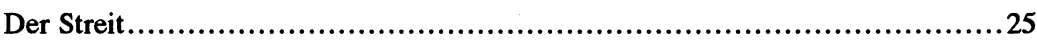

Zum Beispiel: Ein sanftes, lang hingezogenes Ende $25 \cdot$ Schneiders Zorn $27 \cdot$

Aufgeklärte Moderne und melancholischer Regreß? $31 \cdot$ Kein Kronzeuge der

Anti-Melancholie: Peter Sloterdijk 37

Melancholischer Thron und praktische Vermissung - Anmerkungen zu Wolf

Lepenies ...........................................................................41

Figuren der Melancholie......................................................47

Mein Freund Tunda 47. A Man Of About Thirty-Five 50 - Tunda, Flory und

das Warten. Nachgeschobene Gedanken zum Melancholischen 52

3. "Ein wunderbares Gefühl. Ich erinnere mich." Zur melancholischen Befindlichkeit erschöpfter Rebellen ...................................57

Vorbemerkung 57

Lebensweltliche Irritationen ................................................58

Kommunikative Rationalisierung und Selbsterfahrung im Diskurs. 60

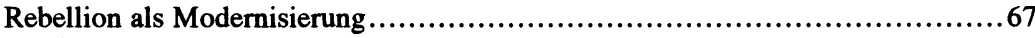

Nicht mehr zauberhaft: Aporien entzauberter Intimität .........................8 81

Zur Vorgeschichte $81 \cdot$ Liebe als Beziehung: Die neuen Konflikte 83

Das Vorspiel hat ein Nachspiel. Acht abschließende Thesen zum 68er-Komplex. ..92

Vorspiel: Protest, Vernunft und neuer Sinn 92 Nachspiel: Unsicherheit - Das

Neue als Bürde 94

4. Verwicklungen und Vermittlungen: Das Medium poetischer Texte und die Lebenswelt .................................................. 101

Visionen einer Erbfolge: Poetik und Poetologie in neuem Glanz ................. 101

Fiktion, Philosophie und Lebenswelt bei Jürgen Habermas....................... 107

Intentionalität poetischer Bedeutungen ..................................... 114

5. Der Taxifahrer.Uwe Timm: Kerbels Flucht .............................. 117

Auflösung des Politischen im verzweifelten Blick............................. 117

Figur, Text, Autor................................................... 124

Heldentum des rebellierenden Körpers und schwacher Trost der Vernunft........ 132

Bilanz ....................................................................... 140 
Sehnsüchtiger Bildersturm - Zur Motivierung der angestrengten Bildlichkeit

Rezensionen und Neubearbeitung 145 - Süchtig nach Bildern 146

Martin: Schichten des Scheiterns

Dort wo man singt 153 • "... und einem Zustand von Gewißheit" 159 • "... auf

dem Abstellgleis" - 164 "...Hand in Hand zu überleben" 167

"Dagegen kam ich nur schreibend an..." .

Martins Hoffnungen 180

7. Der Buchhändler. Botho Strauß: Die Widmung

Was das Normale ist... Zur publizierten Rezeption.

"Man hört nicht zu, man läßt nicht ausreden. Daher macht den Erzähler seine

Gabe verlegen. "Überlegungen zum Erzählmodus der Widmung.

Diffuser Erzähler, starker Autor

"Stille Epoche, die keine Schicksale macht" Über einige Aspekte der

Strauß'schen Geschichtsauffassung

TV als Welt $206 \cdot$ Schlecht gemachte Zeiten? 208 Butter auf dem Kopf 213

"Den toten blauen gottgestohlenen Schimmer, unter dem er schließlich Ruhe fand" 217

Liebe - "Ein altes Wort, man versteht es kaum noch"

Komische Liebe: unangemessen 223 - Finale der Erotik im Zeichen von Permission und Vernunft $225 \cdot$ Gesellschafts- als Textkritik 229

8. Nachbarschaften: Die Journalisten

Vorbemerkung 233

Christoph Meckel: Licht.

Diarische Beschwörung $235 \cdot$ Werbebilder $237 \cdot$ Sauberer Sex $239 \cdot$ Tod: Rettung der Liebe und rettender Ausgang der Erzählung 240

Paul Kersten: Absprung.

Besonderheiten 244 - Unglückliche Liebe und die Legitimationskraft der

Krankheit $244 \cdot$ Streit auf dem Papier: das Alter ego als Briefpartner 247 · Die

Zauberformel der Angst $248 \cdot$ Heimkehr 253

9. Besondere Kennzeichen 257

Zwischen Diarismus und Essayismus

Zeitverschiebungen $257 \cdot$ Muster der Journaux Intimes $259 \cdot$ Geschmack der Wahrhaftigkeit $261 \cdot$ Reflexivität: Der Text gegen den Text $263 \cdot$ Getarnter Essayismus 265

Der Scene verhaftet, aber doch schon fast ein Dichter

Entwicklungslinien 268 Zuschreibungen 279

Im Schmerz geboren: konservativer Protest

Mit den Worten am Ende 289 - Die Frauen: Agentinnen des kalten Fortschritts die Männer: Aristokraten ohne Macht und Geld 292 - Aus der Geschichte fallen 298 


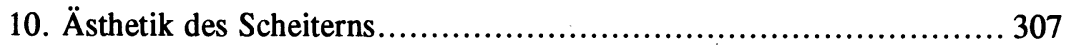

Vom gekränkten Subjekttraum zur imaginären Selbstverwirklichung ............. 307

Subjektdenken, Kränkung, Melancholie 307 · Dissidenzerfahrungen 312

Das literarische Kunstwerk als imaginäre Selbstverwirklichung ................. 314

Von Ohnmacht zu Allmacht 314. Geschichten einer scheiternden romantischpoetischen Selbstfindung 315 - Das imaginäre Wir der Erzählungen: Konturen einer problematischen Selbsterfahrung 323

Metaphysik des verlorenen Glücks: Die romantische ist die wahre Liebe

Neue Sicht: Die Geschehnisse ereignen sich im Inneren des Subjekts

Inszeniertes Leiden, Allegorien und Sprachlosigkeit

Mangel des Lesers $333 \cdot$ Zur Dialektik der Anerkennung 334 $\cdot$ Allegorischer

Zauber 338

Eine letzte Betrachtung: Camouflage des Scheiterns und Vorbereitungen zur

Rückkehr des Dichters

Die Allegorien der scheiternden Helden $344 \cdot$ Allegorien und Leser $345 \cdot$

Rezeptionsverhältnisse $346 \cdot$ Der geborgte Held. Schlußgedanke 349

Verzeichnis der zitierten Literatur: 


\section{Vorbemerkung}

He says: 'There may be a fashion for failure and negation now. But we dont't have to go along with it. ${ }^{\prime}$

'Why not?' asks Barbara, 'after all you've gone along with every other fashion, Howard.'

Malcolm Bradbury: The History Man (1975). London 1984, S.17

Nicht nur den Helden in Bradburys Campus-Novel gerät das Bekenntnis, als Subjekt einer rebellisch gedachten Geschichte gescheitert zu sein, zum festen Topos zeitgemäßer Selbsterklärung. Mitte der siebziger Jahre erklingt aus den intellektuellen Reihen des ehedem revolutionären Zorns zunehmend der sanfte Ton der Resignation. Die Rede vom eigenen Leiden, vom Sinnschwachen der Zeit, von den schmerzhaft glücklichen Ausblicken der Melancholie findet sich rasch auch im aktuellen Repertoire der elitären wie populären Ästhetik. Unzählige Debatten, ausgetragen in den Nischen der Universität, im Scheinwerferlicht der TV-Studios oder am polierten Kneipentisch, analysierten und zementierten das kollektive Gefühl, in eine Zeit der Wende, in rebellische Abschiedsszenen geraten zu sein.

Nicht die politische oder geistesgeschichtliche Bewertung eines Prozesses, der mit dem Zerfall geopolitisch verankerter und Weltbilder prägender Ordnungen nur noch an Unübersichtlichkeit gewonnen hat, bildet das Anliegen des vorliegenden Bandes. Sein Thema ist vielmehr ein Phänomen, das eng mit der wechselhaften Rolle des Poetischen als eines gesellschaftlichen Mediums der Wahrheits- und Erfahrungssuche zusammenhängt: Wie ist es möglich, daß sich in Akten ästhetischer Deutung und Aneignung eine Erfahrung des Scheiterns in ein Bewußtsein eigensinniger Überlegenheit verwandelt?

Auf diese Frage stößt unweigerlich der Leser jener ab der Mitte der siebziger Jahre zuhauf erscheinenden Erzähltexte, deren Geschichten die Verwerfungen eines Subjekts bilden, dem die Erinnerungen an bessere Tage und mithin auch die Vorstellungen von Zukunft entfliehen. Obschon von der zeitgenössischen Kritik gelegentlich als Zeugnis einer Neuen Weinerlichkeit abgestempelt, inszenieren die Erzählungen nicht lediglich schlichte Kläglichkeit. Selbst die Tode, die die Helden jener Fiktionen zu sterben haben, umgibt ein diffuser Glanz des Heroischen und des in praktischen Akten schwerlich einzuholenden Triumphs.

So liest man beständig vom noch jungen Mann, dem mit dem Verlust seiner Liebe auch ein bislang für real geglaubter Sinn abhanden kommt. Was ihm die Gesellschaft der Freunde anrät, schlägt er aus. Seiner Biographie nach ein Kind der späten sechziger Jahre, verweigert er, was die emanzipatorische Aufklärung als Rettung offeriert: Trauerarbeit und Ablösung. Er nimmt das Erlebnis der verblichenen Liebe als Schlüsselerfahrung über die ihm eigene Welt und läßt fast jede Hoffnung, sich aus der Hölle der Schmerzen befreien zu können, fahren. 
Der geschlagene Mann begibt sich in die Klausur seines Leidens und setzt zu einem, bei Lichte betrachtet, merkwürdigen Projekt an. Er widmet seine traurigen Stunden dem Papier. Was er diesem anvertraut, handelt nicht allein vom Individuellen seines Schmerzes. Vielmehr nimmt er Revanche an einer Welt, die seinem Traum vom Großen Sinn abhold geblieben ist. Für wen er zuweilen kunstfertig, häufig essayistisch sein Wissen entfaltet, bleibt lange ungewiß, und endlich teilen die Notate das Schicksal ihres fiktiven Autors: die schönen Sätze landen auf dem Müll. Indes, was sich innerhalb der Geschichten ereignet, vollzieht sich in der realen Lektüre der Prosa nicht. Als reale Leser dürfen wir schließlich goutieren, wovon die Welt der Helden vermeintlich nichts wissen will. Gleichsam aber ist die soziale Sphäre, in der sich die Erzählungen bewegen, jener des Lesers in bemerkenswerter Weise anverwandt. Was man als Trick der Fiktionen begreifen könnte, verweist unter analytischen Aspekten darauf, wie inhaltsästhetische Konstellationen und Ansprüche des poetischen $\mathrm{Me}$ diums zusammenspielen. Erst unter einem erweiterten Blickwinkel zeigt sich, daß die melancholischen Traktate und die sie umgebenden Fiktionen einen Diskurs beherbergen, dessen Intentionen einem veränderten sozialen Verständnis des Dichters gelten.

Bei den nachfolgenden Kapiteln handelt es sich um eine überarbeitete und gekürzte Fassung meiner 1990 vom Fachbereich Sprachwissenschaften der Universität Hamburg angenommenen Dissertation. Die dort vorgeschlagenen Überlegungen wenden sich nicht an ein transhistorisches Reich der Dichtung, sondern versuchen einer sozialgeschichtlich oder sozialwissenschaftlich interessierten Betrachtungsweise die Treue zu halten. Zum ästhetischen Gegenstand soll also eine Haltung der Distanz gewahrt werden. Gleichwohl hat mich kein esoterisch akademisches Interesse auf die Texte Theobaldys, Strauß', Kerstens oder Timms stoßen lassen. Vielmehr handelte es sich bei meiner ersten Lektüre auch um eine, die am eigenen Leib erlebt geglaubte Erfahrungen in den Erzählungen wiederzufinden meinte. Doch bereits die ersten Versuche, jene Erfahrungen aus ihrer narrativen Umgebung zu lösen und zur verallgemeinernden Sprache zu bringen, zeigten, im Dunstkreis von Aphorismen und essayistischen Notaten verharrend, welchen Täuschungen eine Lektüre aufsitzen muß, die den sozialen Spuren der poetischen Inszenierung keine Beachtung schenkt. Was aber heißt es, sich einer Haltung der Distanz gegenüber den - schließlich eigenen - Erlebnissen der Lektüre zu unterwerfen? Dies bedeutet zum Beispiel, sich in einem Apriori der Suggestion einer subjektiven Offenbarung zu verweigern, die auf das Absolute oder Existentielle ihrer Konstruktionen zielt. So gilt es, über die geschichtliche Konsistenz jenes Großen Sinns, dessen soziale Abwesenheit die postrebellischen Erzählungen als Erfahrungen ihrer Zeit reklamieren, nachzudenken. Und es geht etwa auch darum, die Rolle zu ergründen, die die Klage über den Verfall einer Liebesbeziehung für die inhaltsästhetischen wie konstruktiven Momente der Fiktionen in Abhängigkeit eines gesellschaftlichen Diskurses spielen.

Gleichwohl steht uns keine historische Konstruktion zur Verfügung, die den Interpreten gewissermaßen in eine Position außerhalb jener Wirklichkeit rückt, in welcher die poetischen Texte ihre Bedeutungen entfalten. So wäre es geradezu absurd, der Annahme anzuhängen, in einem Reich der Theorie all jene Fragen zu lösen, derer sich die poetischen Texte mit all ihrem ideologischen Ballast an- 
nehmen. Wie es sich um die Beziehung zwischen den Medien der sozialwissenschaftlichen Theorie und des Poetischen verhalten mag, versuche ich im 4 . Kapitel am Beispiel einer aktuellen Kontroverse $\mathrm{zu}$ ergründen. Auf die Darstellungen in diesem Kapitel sei auch verwiesen, wer vorab nähere Informationen zu den methodischen Strategien dieser Arbeit sucht. Nicht mit einer methodologischen Reflexion setzt die Arbeit ein, sondern mit der Reflexion von Begriffen und Motiven, die die Diskurse um die neuen Zeiten nach dem Protest begleiten: Sinn, Sinnkrise, Melancholie, Rationalisierung.

Im Zentrum des 1. Kapitel steht eine Auseinandersetzung mit dem in den spekulativen Zeitdiagnosen häufig strapazierten Begriff des Sinns. Vorgeschlagen wird eine theoretische Einstellung zum Gegenstand, die der jeweiligen Rede von dem einen Sinn zuwiderläuft. In Anschluß an die Diskussion eines theoretischen Konzepts vom Sinn werde ich unter anderem darlegen, wie man die Klage über die Sinnlosigkeit auch verstehen kann.

Das 2. Kapitel bringt ästhetische Fragen deutlicher ins Spiel. Eine Beschäftigung mit der zeitgenössischen Kulturkritik rückt den Streit um die Rechte der Melancholie in den Blickpunkt und mündet in dem Versuch, eine im weitesten Sinne heuristische Idee vom Melancholischen zu gewinnen.

Die zeitgenössischen Motive der Melancholiedebatte greift das 3. Kapitel zum Katzenjammer der siebziger Jahre auf. Hier bemühe ich mich, die bislang verfolgten Fäden zu verbinden und Thesen zur Bewußtseinslage jener Szene vorzulegen, die das Personal, die Leserschaft und die Autoren der literarischen Texte verbindet. Thesen, die vorschlagen, den Protest von 1968 und die jeweils subjektive Erfahrung seines Zerfalls im Zusammenhang eines Komplexes kommunikativer Rationalisierung (Habermas) zu sehen, beschließen den historischen Versuch.

Das bereits erwähnte 4. Kapitel debattiert methodologische Fragen der Literaturwissenschaft und erörtert den Anspruch, den Poetik und Literaturkritik aktuell reklamieren dürfen. Indirekt wird auf diese Weise der Rahmen abgesteckt, in dem sich die nachfolgenden Interpretationen bewegen.

Im 5. bis 8. Kapitel folgen die eigentlichen Textstudien. Dabei handelt es sich um prinzipiell eigenständige Untersuchungen zu Erzählungen, deren Verwandtschaft ich in einem methodischen Vorgriff behaupte. Das 8. Kapitel vertieft die Thesen zur Homogenität einer signifikanten Mehrzahl von Texten: Den ausführlicheren Interpretationen von Timms, Theobaldys und Strauß' Erzählungen werden zwei knapper gehaltene, exemplarische Lektürevorschläge zu Texten nachgestellt, die auf den ersten Blick vielleicht randständig anmuten.

Das 9. Kapitel geht den konstruktiven Hintergründen der diagnostizierten Verwandtschaft nach. In den Blick genommen werden die Funktion der diarischen Techniken und die Affinität zu essayistischen Literaturformen. Zudem wird überlegt, welche Funktion der Liebesepisode in allen Texten für die Motivation einer konservativen und melancholischen Weltdeutung zukommt.

Das 10. Kapitel greift diese Überlegungen auf und stellt sie in einen kulturgeschichtlichen Zusammenhang, der einerseits die Argumente des sozialhistorischen Teils einbringt, andererseits aber auch poetologische Thesen berücksichtigt, deren Thematik über den bislang thematisierten Textkorpus hinausreicht. In kritischer Nähe zu Peter Bürgers Lesart moderner Prosa betrachte ich die Erzäh- 
lungen als Produkte einer imaginären Selbstverwirklichung und mithin als Unternehmungen, die in enttäuschten Subjektträumen gründen. Die Beziehungen zwischen Leser und Text, zwischen Autor und Publikum werden schließlich im Rückgriff auf Walter Benjamins Verständnis der Allegorie betrachtet. Als Ergebnis dieser Überlegungen erhärtet sich eine bereits im 9. Kapitel skizzierte These, die ein verändertes Verständnis des sogenannten Neuen Subjektivismus nahelegt.

Für die langwährende Arbeit an meinem Projekt schulde ich manchen Dank. So bin ich in wesentlichen Phasen von der Friedrich-Naumann-Stiftung finanziell unterstützt worden, und auch meine vom Deutschen Akademischen Austauschdienst (DAAD) geförderte Tätigkeit in England ließ mir für wichtige Vorarbeiten Zeit. Für die anregende Betreuung meiner Arbeit danke ich Prof.Dr. Ludwig Fischer, aber auch den informellen Kritikern sowie der zweiten Gutachterin, Prof.Dr. Marianne Schuller. Nicht zuletzt gilt mein Dank den MitarbeiterInnen des Rhoner Kreises. 